\title{
UNA MIRADA A LA CIENCIA POLÍTICA EN HONDURAS: LA NECESIDAD DE SENTAR BASES PARA SU INSTITUCIONALIZACIÓN
}

\author{
Álvaro Cálix \\ FundaCIÓN FrIEDRICH EBERT, HONDURAS \\ ROLANDO SIERRA \\ Centro de Investigación y Promoción de los Derechos Humanos, Honduras
}

\begin{abstract}
Resumen
El presente artículo tiene como propósito esbozar, a groso modo, el contexto, los antecedentes, los alcances y los desafíos de la ciencia política en Honduras. A priori, se reconoce en el país un rezago significativo de esta disciplina dentro del campo de las ciencias sociales. Tal aseveración causa mayor preocupación si se observa que el derecho, la sociología, la historia y la psicología -que aparecen como ciencias con mayor despegue relativo en Honduras, ya que cuentan con una mayor institucionalización académica-, no han alcanzado niveles de consolidación adecuada por la escasa acumulación, producción y actualización del conocimiento científico. Aun así, en los últimos cinco años han surgido en el país iniciativas para propiciar un mayor desarrollo de la Ciencia Política, que a pesar de una cierta dispersión de los esfuerzos, ha generado un renovado interés por este campo del conocimiento.
\end{abstract}

\begin{abstract}
The purpose of this article is to highlight, grosso modo, the background, context, advances and challenges of political science in Honduras. A priori, one has to acknowledge the meaningful setback of this discipline within the field of social sciences. Such an assertion causes great concern if it is considered that the juridical, sociological, historical and psychological sciences, - that appear as sciences with a relative high development in Honduras due to a strong academic institutionalization-, have not reached the right levels of consolidation due to the scarce accumulation, production and update of the scientific knowledge. Nonetheless, during the last five years some initiatives have emerged in the country to propitiate a higher development in political science, which in spite of a certain dispersion of efforts, has generated a renewed interest for this field of the knowledge.
\end{abstract}

PALABRAS CLAVE • Ciencia Política • Honduras • Historia de la Disciplina • Institucionalización Académica

\section{ALGUNOS ANTECEDENTES EN LA EVOLUCIÓN DEL PENSAMIENTO Y EL CONOCIMIEN- TO POLÍTICO}

Es hacia finales del Siglo XIX, cuando se puede advertir en Honduras el inicio de una preocupación más o menos consistente por la construcción de un pensamiento político que explique las dificultades de estructurar un Estado republicano en un territorio fragmentado y con escasa cohesión social. Tal preocupación tiene lugar en el contexto de los intentos de modernizar al país al impulso de una serie de medidas enmarcadas dentro del proceso de la reforma liberal. 
Ahora bien, el contenido de este pensamiento que va surgiendo desde aquella época no necesariamente se decanta por un enfoque cientificista, más calza con el ensayo y la opinión, aunque, valga decirlo, ello no obsta para advertir ensayos y estudios sobresalientes respecto de la sociedad hondureña en clave política, los cuales han contribuido a una mayor compresión y análisis de la dinámica y racionalidad de los actores políticos.

Cabe advertir que el contexto histórico en que se desarrolla el estudio de la política en Honduras se caracteriza por un sistema político-institucional débil, deudor de una matriz autoritaria y patrimonialista, en el que los procesos de democratización y modernización en general se han visto constantemente truncados. En efecto, el régimen político hondureño arrastra una manifiesta debilidad del Estado de Derecho y subiste una importante brecha de participación entre la clase política y la ciudadanía, esto desde la creación de la República, pasando por la reforma liberal del siglo XIX, las guerras civiles de la primera mitad del siglo, la dictadura de Carías (1933-1949), los procesos reformistas de esta segunda mitad de siglo bajo los gobiernos de Gálvez y Villeda y el militarismo de 1963 a 1980. Salvando una que otra excepción, el principal legado de estos regímenes políticos fue la inestabilidad política, la debilidad institucional, el clientelismo y una ciudadanía permanentemente reducida y, en buena medida, ficticia. Sin embargo, a veinticinco años de haber comenzado el actual proceso de democratización (1980-2005), han surgido algunas condiciones favorables, aunque no capitalizadas aún, para el despegue definitivo de la disciplina.

Bien, dentro de ese contexto histórico, la evolución de la ciencia política en Honduras presenta un proceso de institucionalización en el ámbito académico sólo en las últimas décadas, acompañado del surgimiento y avance de otras disciplinas en el campo de las ciencias sociales que han contribuido al estudio del fenómeno político, especialmente la historia y la sociología.

Al influjo de las ideas positivistas de Europa, un vistazo a las décadas finales del siglo XX refleja que existió una iniciativa en 1882, bajo el impulso del político e intelectual Ramón Rosa, que se concretó en la fundación de la Facultad de Jurisprudencia y de Ciencias Políticas, con una clara intención de introducir y desarrollar en el país el abordaje científico de la política. No obstante, esta iniciativa no tuvo el seguimiento adecuado, por lo que el peso de la rama jurídica terminó por absorber a dicha Facultad, de manera que en 1947 se le cambió el nombre, pasándose a llamar Facultad de Ciencias Jurídicas y Sociales, relegándose la enseñanza de la política a asignaturas de derecho político, economía política, sociología, filosofía del derecho y la teoría del Estado. La enseñanza de las ciencias políticas como asignatura es retomada por la Facultad de Ciencias Económicas en 1950 y por el Centro Universitario de Estudios Generales (CUEG) a partir de 1961, dentro de la Universidad Nacional Autónoma de Honduras (UNAH) (Paz, 2004 y 2005).

En 1978, la reforma universitaria de ese año suprimió las asignaturas generales de sociología y ciencias políticas y fueron sustituidas por las asignaturas de realidad socioeconómica de Honduras y teoría general de Estado y del Derecho. Pero en 1982 se restauró la antigua nomenclatura de sociología, ciencias políticas. La asignatura de sociología quedó a partir de ese momento como una materia obligatoria en todas las carreras impartidas por la UNAH, mientras que la de ciencias políticas es sólo obligatoria en las carreras comprendidas en las ciencias sociales.

Hoy día, uno de los mayores problemas es el hecho de que en Honduras aún no existen estudios de ciencia política a nivel de licenciatura. Pese a esa ausencia, desde los años 90 del siglo XX, se 
han impulsado en la UNAH dos proyectos de creación de maestrías en ciencia política: La primera viene desde la iniciativa de la Facultad de Derecho y la segunda comenzó desde el Departamento de Ciencias Sociales. La primera iniciativa aún no ha podido comenzar a andar, en tanto que la segunda inició en el año 2004 la primera promoción, con dos grupos simultáneos en las ciudades de Tegucigalpa y San Pedro Sula, en el marco del convenio internacional de cooperación científico-técnico firmado entre la UNAH y la Universidad de La Habana (Paz, 2004)1.

Aunque ahora se observe un leve proceso de institucionalización de los estudios políticos en el país, puede decirse, que en los últimos 25 años los avances mayores en el campo académico se observan en el área de la sociología política, gracias al aporte de un grupo de sociólogos, entre los que sobresalen Mario Posas, Leticia Salomón y Julieta Castellanos. De los tres, L. Salomón, es la que prácticamente desde 1980 ha mantenido una producción sostenida dentro de los estudios de la sociología política al menos en tres temas de estudio: el papel de los militares en la vida política del país, el proceso de transición a la democracia y la construcción de ciudadanía, y la dinámica de la sociedad civil.

Con la paulatina subordinación del poder militar al poder civil, se va dando un viraje en los estudios políticos respecto a los primeros años de la transición. De los estudios sobre las relaciones cívicomilitares se ha ido pasando a nuevas preocupaciones asociadas al diseño institucional que requiere el Estado democrático de derecho, así como sobre el papel de la sociedad civil y los partidos políticos en la profundización del régimen. También ha surgido desde los años 90 un renovado interés en las posibilidades del proceso de descentralización y desarrollo de los gobiernos locales.

De una u otra forma la reforma política en estos veinticinco años ha estimulado el análisis sobre el proceso de transición. Aun así, cierta fractura en la identificación de objetos de estudio propios de la ciencia política, influye junto a otros factores para que hasta la fecha se carezca de trabajos en profundidad que aborden de forma holística el sistema político del país. Un déficit particular se observa en la falta de investigaciones sobre el sistema de partidos. Por diversas razones los partidos políticos sólo han sido tangencialmente abordados desde el mundo académico hondureño, y muy poco desde un ángulo estrictamente científico.

Este rápido vistazo a los antecedentes permite señalar que la ciencia política en Honduras tiene el desafío de la formación de profesionales en este campo así como de su institucionalización en general, y sobre todo de la creación de grupos permanentes de pensamiento e investigación.

\section{FORTALEZAS, OPORTUNIDADES Y DESAFÍOS}

Una de las principales fortalezas para la disciplina sea quizás el hecho que en los últimos diez años se ha generado un interés en ir propiciando espacios de formación académica a nivel universitario, que ha implicado el surgimiento de iniciativas para crear dos maestrías relacionadas para

1 La Maestría en Estudios Sociales y Políticos está orientada más a la comprensión del fenómeno político desde un enfoque transdisciplinario de las ciencias sociales, mientras que la otra maestría estará un poco más perneada por el enfoque jurídico. Los estudiantes de la actual (primera) promoción de la Maestría en Estudios Sociales y Políticos proceden de diversas carreras, aunque se observa una proporción notable de profesionales con licenciatura en periodismo o ciencias de la comunicación y docentes universitarios que imparten la asignatura de Ciencias Políticas u otras materias del departamento de Ciencias Sociales de la UNAH. 
estudiar el fenómeno de la política, lo cual es un punto de referencia para la institucionalización y profesionalización de esta disciplina.

Por otra parte existe un número reducido pero significativo de hondureños que han salido del país para emprender especializaciones relacionadas con el estudio de la política. Algunos han estudiado en Estados Unidos, Inglaterra, y otros en Sudamérica. Especial mención merece la Maestría Latinoamericana de Estudios Sociopolíticos de la Universidad Alberto Hurtado de Chile, que se ha convertido en un referente en la última década, y al menos cinco hondureños se han graduado en dicha maestría en esa etapa.

No podría desconocerse como oportunidad el hecho de que varias agencias de cooperación internacional, directa o indirectamente, han venido apoyando ciertos estudios y procesos relacionados con dimensiones de la política, en clave de profundización del régimen democrático. Dentro de ese espectro se inscribe el auspicio de la cooperación sueca (ASDI) y la coordinación del PNUD $^{2}$ en temas concernientes al proceso de reforma política electoral que se ha observado entre 2001 y 2005 -que incluye la realización de varios diagnósticos y estudios de percepción sobre el pensamiento de las elites y de la ciudadanía en general sobre el quehacer político y las reformas electorales ${ }^{3}$-. Asimismo, la USAID ha apoyado estudios sobre el fortalecimiento del poder Judicial, la administración electoral y, de manera particular, sobre la cultura política; y el Banco Mundial en el análisis de la corrupción y sus implicaciones para la legitimidad del Estado.

Por otra parte la cooperación española (AECI) tiene varios años de estar impulsando estudios e intervenciones en el campo del desarrollo municipal, la descentralización y el fortalecimiento del sistema de justicia; la OEA se ha visto involucrada en estudios sobre la situación y papel de los partidos políticos, incluyendo el análisis y potenciación de la participación de la juventud en los partidos, en tanto que la Unión Europea (UE) ha apoyado procesos para potenciar las condiciones de la participación ciudadana en los asuntos públicos. En el campo del debate destaca el papel que ha jugado la Fundación Friedrich Ebert en propiciar espacios de diálogo y debate sobre los retos del quehacer político en Honduras, produciendo además una serie de boletines que sitúan temas coyunturales acerca de la dinámica de los actores políticos.

A un nivel más propiamente académico se encuentran algunos estudios realizados por el Instituto Centroamericano de Estudios Políticos (INCEP), con sede en Guatemala, y que apoyado por la fundación Konrad Adenauer, ha realizado estudios políticos comparados en varios países centroamericanos. También se puede mencionar las investigaciones regionales de FLACSO en el área de gobernabilidad, municipalismo y seguridad ciudadana, así como los estudios puntuales del Instituto de Estudios de Iberoamérica y Portugal -adscrito a la Universidad de Salamanca-, bajo la iniciativa del Dr. Manuel Alcántara, particularmente en el estudio del funcionamiento del Congreso Nacional, sistema de partidos y funcionamiento del sistema de justicia. Más recientemente, desde 2004, la antena para Centroamérica del Instituto de Altos Estudios para América Latina (IHEAL),

2 ASDI y el PNUD también han tenido un activo apoyo al estudio y fomento de las condiciones para la participación política de las mujeres. EI PNUD también apoya en la realización de estudios y proyectos tendientes a mejorar la gobernabilidad democrática, mediante diagnósticos y acciones para mejorar la interacción de los operadores de justicia, y ha impulsado un proceso para la redefinición de una política de defensa dentro de la concepción de la seguridad democrática.

3 Sobre la administración de procesos electorales, en los años ochenta y noventa fue crucial el apoyo brindado por el Instituto Interamericano de Derechos Humanos (IIDH), con sede en Costa Rica, mediante el Centro de Asesoría y Promoción Electoral (CAPEL). 
adscrito a la Universidad de la Sorbona, desde su sede en Guatemala está impulsando una red de politólogos que construyan y desarrollen una agenda de investigación en el campo de la geografía política, campo apenas explorado en la sub-región. Tampoco podría dejar de mencionarse el convenio de la Universidad de la Habana para apoyar la Maestría en Estudios Políticos y Sociales que imparte la UNAH y los estudios que el Dr. Mitchell A. Seligson -ex investigador de la Universidad de Pittsburg (ahora en la Universidad de Vanderbilt)- ha llevado a cabo en Centroamérica, incluyendo a Honduras, sobre temas atinentes a la cultura política.

Pese a reconocer la valiosa oportunidad que representan las iniciativas antes citadas, la mayoría de ellas suelen carecer de una perspectiva de largo plazo que consolide las capacidades de investigación politológica en el país. En adición, la mayor parte de los estudios son realizados por consultores e investigadores extranjeros, con una participación más reducida de investigadores nacionales. Por otra parte la divulgación de los estudios no logra superar muchas veces el lanzamiento de los reportes de investigación, y es que en general no existe en el país una consistente absorción científica para debatir y plantearse nuevas preguntas de investigación a partir de esos estudios financiados con fondos externos.

En vista de que el país no cuenta siquiera con una carrera de pregrado en ciencia política a nivel universitario, resulta evidente que uno de los desafíos prioritarios es la institucionalización académica de la misma. Más allá de que exista un grupo de hondureños(as) cursando en el extranjero estudios politológicos o afines, no se distingue una comunidad de cientistas políticos -ni siquiera existe un registro para saber cuántos son, mucho menos algún tipo de asociación-que pretendan como proyecto la fundación de los cimientos que permitan un desarrollo consistente de esta ciencia en Honduras ${ }^{4}$.

Es decir no se está generando la experiencia que ocurrió con la sociología. En efecto, fue a partir de 1990 cuando la UNAH abrió la carrera de sociología, debido en buena parte a un grupo de jóvenes profesionales que en los 70 y 80 fueron a estudiar sociología a la Universidad de Costa Rica y que se convirtieron la mayoría de ellos en docentes en la UNAH. Y aunque la carrera no ha despegado como se pretendió en un primer momento, al menos se ha mantenido y cuenta ya con varias promociones de graduados.

Como se mencionó antes, frente a esa ausencia de bases académicas en el nivel universitario, en las ultimas tres décadas el análisis politológico prácticamente ha dependido de profesionales de otras ciencias sociales. De profesionales de la ciencia jurídica antes de los ochenta y, después de esa década, ya más a cargo de sociólogos e historiadores que se han avenido a las orillas de esta disciplina. La carencia de una carrera universitaria ha influido además en la ausencia de una institucionalización de la labor investigativa universitaria y, por ende también, de la divulgación y la discusión científica en esta rama; lo cual redunda en que se adolezca de una comunidad científica politológica. De ahí que en el país no exista ningún congreso de ciencias políticas ni publicaciones periódicas especializadas. Asimismo, conviene señalar que ninguna de las siete universidades privadas que funcionan a la fecha tiene cabida para la ciencia política y, en general, para las ciencias sociales, por cuanto se han enfocado preferentemente en la administración de empresas y negocios, turismo e ingenierías. Como excepción en las universidades privadas, la Universidad

4 Aunque no se cuenta con un registro formal, se estima que en el país no existen más de diez hondureño(as) que cuenten con estudios de doctorado en ciencia política. 
Católica ha creado la carrera de Derecho, impartiéndose en dicha carrera algunas materias relacionadas con la ciencia política. Conviene apuntar que dentro de la otra universidad pública -La Universidad Pedagógica-, se imparte la asignatura de ciencias políticas dentro de la carrera de ciencias sociales.

Relacionado con lo anterior, también se identifica la necesidad de individualizar el perfil de la ciencia política en Honduras, y dejar de depender sólo de las aproximaciones que algunos destacados profesionales han hecho desde el campo de la historia y la sociología política. Esto no significa romper los vínculos con dichas disciplinas pero sí desarrollar cierta autonomía que le permita definir y aplicar objetos de estudio y metodologías propias de la disciplina.

Si bien es una fortaleza la existencia de las dos iniciativas de maestrías en la UNAH relacionadas con la ciencia política (mencionadas en el acápite anterior), existe un vacío por la falta y sustentación de base a nivel de pregrado, y no solamente como una asignatura de estudios generales que deben cursar los estudiantes de las carreras de ciencias sociales impartidas por la UNAH. Desde un punto de vista crítico y de maximización de esfuerzos, podría reprocharse el hecho de que se pretenda contar con dos niveles de especialización en ciencia política en la misma universidad pero sin que se hayan aunado iniciativas para crear la licenciatura.

Respecto al campo temático, otro desafío tiene que ver con la necesidad de identificar objetos de estudio acotados y relevantes para el país que dé paso a la construcción de agendas y programas de investigación sobre temas aún ausentes de tratamiento científico. Existen estudios y ensayos sobre la transición política a la democracia comenzada a inicios de los años 80 del siglo pasado, también algunos trabajos importantes sobre las relaciones entre el poder militar y el poder civil, sobre la dinámica y el papel de la sociedad civil, pero prácticamente no existe mayor logro en estudios profundos sobre el sistema de partidos ni sobre las relaciones entre los poderes del Estado.

Un desafío particular consiste en la urgencia de contar con espacios divulgativos sobre la producción generada en el país, que aunque escasa, no cuenta además con los estímulos de publicación, no solo bajo el formato de libros, sino que tampoco en revistas o boletines de ciencia política. Además, es notable la ausencia de congresos, foros y debates sobre bases de argumentación científica.

En lo que atañe al campo laboral, se aprecia que no existe una demanda formal de politólogos de carrera para la asesoría política de los congresistas, candidatos a cargos de elección, Poder Ejecutivo, Agencias de Cooperación Internacional y no digamos la Empresa Privada. Esto quizás tenga que ver con el hecho que al politólogo tiende a vérsele como un tipo de intelectual que hace análisis político como una actividad ocasional o como un área de desarrollo laboral no exclusiva.

En al área de los estudios de opinión política se advierte que el mercado ha sido absorbido más por los expertos en marketing, sin que necesariamente se realice un análisis político a profundidad a partir de los sondeos de opinión. De manera que como oportunidad de trabajo, la coyuntura electoral hace que se demande más a aquellas firmas con capacidad de hacer encuestas y creación de imagen de los candidatos y partidos, en desmedro del análisis político propiamente dicho. 
Así la situación, el campo laboral desarrollado para y por los politólogos es reducido. No es la docencia el predominante, porque la mayoría de los profesores que sirven la clase de ciencia política, y afines, vienen del campo del derecho o de la sociología ${ }^{5}$. En la maestría en estudios políticos y sociales, debido a la ausencia de profesionales con grado de Doctorado en el campo, los catedráticos suelen ser profesores visitantes que provienen de Cuba y en menor medida de los Estados Unidos. Por otra parte, algunos profesionales avenidos al campo de la política encuentran ciertos espacios dentro de las ONG, requeridos no tanto para la investigación y el análisis político sistémico, sino más bien por sus habilidades en gestión de proyectos y capacidad de echar a andar procesos de incidencia política. Otros incursionan en el periodismo y unos menos en la mercadotecnia política. Muy pocos han figurado como asesores a políticos; y como se dijo antes, ni siquiera en el propio Congreso Nacional se cuenta con un cuerpo asesor de politólogos, mucho menos los propios partidos, el Poder Ejecutivo ni los órganos de justicia y administración electoral ${ }^{6}$. En lo que se refiere al sector privado, en general no hay una demanda formal de profesionales de la ciencia política.

\section{LAS PREGUNTAS DE INVESTIGACIÓN: ¿QUÉ PREOCUPACIONES HAN ESPOLEADO EL ESTUDIO DE LA POLITICA EN HONDURAS?}

No sería antojadizo suponer que la ausencia de institucionalidad de la ciencia política en el país ha influido en el tipo y profundidad de las preguntas de investigación surgidas. En los parámetros del fenómeno político, para el caso hondureño, de alguna manera ha existido una mayor preocupación por indagar acerca de la praxis política, y una menor atención al análisis estructural de ésta.

Antes de la recuperación democrática de los 80 (en las décadas del 60 y del 70), el abordaje de la política en Honduras parecía oscilar entre un enfoque conservador normativo -desde el lente del derecho político- y un enfoque dogmático de cariz contestatario, y hasta cierto punto, tributario de metaenfoques regionales como el de la tesis de la dependencia, que aunque de núcleo economicista, logró en su momento pernear el análisis social y político en varios países latinoamericanos, entre ellos Honduras. Y esta polarización se concreta en las orientaciones de la acción en uno y otro grupo. En el primero, escoltado por los abogados, prevalecía el análisis jurídicoinstitucional y la evocación de los derechos de ciudadanía, principalmente los civiles y políticos; mientras que el segundo privilegiaba el análisis de la coyuntura política, la comprensión política de la lucha de clases y la formación de cuadros partidarios para diseñar estrategias contrahegemónicas ${ }^{7}$. Sin perjuicio de la racionalidad y legitimidad de cada una de estas tendencias, lo

5 El salario de los profesores universitarios en la UNAH varía según el nivel que el catedrático ocupe en el escalafón. Los hay profesores que imparten una sola clase, con un tipo de contrato por hora, que devengan al mes un aproximado de US\$ 200; los profesores que tienen contrato mediante Acuerdo, con una carga académica de tres a cuatro asignaturas reciben un aproximado mensual US\$1.200, según el grado y especialización académica del docente. Profesores en la categoría más alta de remuneración, dedicados a tiempo completo, pueden llegar a devengar entre US\$2.500 a US\$3.000. LoS catedráticos que desempeñan algún cargo de dirección académico-administrativa gozan de un Plus a su salario normal. La remuneración en las universidades privadas suele ser un tanto menor a la que ofrece la UNAH, pero la principal diferencia es que los beneficios sociales que otorga la UNAH difícilmente pueden ser concedidos en los centros privados.

6 La función de análisis político al interior de estas instituciones ha estado a cargo de abogados, sociólogos, y en más de algún caso por economistas avenidos a la política.

7 Esta corriente contestataria tuvo como ventana de oportunidad de formación las becas que varias decenas de hondureños obtuvieron para ir a realizar estudios de profesionalización política en la Escuela Nico López de la Habana, la Escuela de Cuadros del PCUS en Moscú y la Academia de Ciencias Políticas y Gestión Social en Sofía (Orellana, 2005). 
cierto es que de cara a la acumulación de conocimiento científico, el producto fue más bien residual, aparte de marginal.

Durante los 80, en cambio, el estudio de la política estuvo fuertemente influenciado por la descripción y análisis de la transición democrática, no tanto desde el punto de vista juridicista, sino más bien desde la perspectiva sociológica e histórica. Varios de los más sobresalientes analistas políticos del país tuvieron como referente la descripción y explicación de la transición centroamericana que elaboró el sociólogo guatemalteco Edelberto Torres Rivas, en especial durante la permanencia de éste como docente de la Universidad de Costa Rica. Lo anterior da pie para insinuar que la mirada política a las democracias emergentes en Latinoamérica, no abrevaba directamente de las vertientes teóricas que analizaban las democracias del mundo occidental desarrollado. Lo cual tiene mucho sentido, si se advierte que las democracias consolidadas se problematizaban en torno al tema de la gobernabilidad, a partir de una lectura que reconocía un exceso de demandas ciudadanas que ponía en riesgo la legitimidad del régimen. En cambio, en la mayor parte de Latinoamérica el reto era construir ciudadanía y cimientos fuertes del Estado de Derecho, claro está, en el contexto de una situación económica en general desfavorable.

Los planteamientos de politólogos como Lechner y O'Donnell, de alcance regional, llegaban a Honduras procesados desde la Sociología Política para intentar observar y comprender los problemas y desafíos de la reciente democratización. Ya en los años 90, especialmente desde la segunda mitad de ese decenio, se advierte un debate, aunque con bajo perfil científico-académico, sobre si el país ha culminado o no la etapa de transición. El debate ha girado en derredor de posiciones que afirman que se ha afianzado la democracia por la ocurrencia consecutiva de siete elecciones generales y por la subordinación significativa del poder militar al poder civil. Otros, en contrapunto, indican que esos aspectos no son suficientes para asegurar que la democracia hondureña está ahora en un proceso de consolidación, pues señalan que una democracia que no ha sido catalizadora de un cambio social que permita sacar a tres cuartas partes de la población de la pobreza, no ha entrado en una fase de estabilidad, y ahora plantean que el incremento de los niveles de violencia y delito están destruyendo más la cohesión social y a su vez están siendo justificación para adoptar medidas de corte represivo que pueden hacer retroceder el conjunto de libertades civiles que la democracia ha permitido hasta ahora.

Siempre en sintonía con la discusión inacabada sobre el resultado de la transición democrática, han emergido nuevas preocupaciones que superan la reflexión acerca de la continuidad electoral. Uno de los aspectos que subyace en el análisis político es la reflexión y la crítica a la corporativización del Estado a cargo de instancias privadas que influyen con pocos márgenes de transparencia en la toma de decisiones gubernamentales. De ahí que comienza a tomar fuerza el análisis sobre el poder y naturaleza de los poderes fácticos en Honduras.

Asimismo, se aprecia ya una cierta alarma sobre una posible erosión de la legitimidad del sistema bipartidista real que prevalece en el país, con dos de los partidos más antiguos del continente y que pese a no haber realizado reformas sustanciales al interior de los mismos, sí se han actualizado electoralmente para captar un significativo voto duro que los sitúa como las fuerzas políticas de mayor convocatoria. Los que postulan tal erosión arguyen que se observan síntomas de ese fenómeno en la creciente sensación de malestar con el desempeño de ambos partidos cuando ejercen el poder público, aparejado al incremento de demandas sociales insatisfechas. 
Desde luego que existen otras preguntas en liza, además de las anteriores, tales como la preocupación por fortalecer las relaciones entre sociedad civil y Estado, la ampliación y profundización de los mecanismos de participación ciudadana (con énfasis en la auditoria social y rendición de cuentas) y la independencia de los poderes del Estado (especialmente del Poder Judicial); sin embargo, una peculiaridad de estas inquietudes es que las reflexiones suelen traducirse en abordajes fraccionados y de corto alcance. No parece haber mayor preocupación -y esto tiene ventajas pero también inconvenientes- por englobar el análisis bajo la síntesis ordenadora de teorías integradoras que den cuenta del fenómeno político desde el nivel estructural. De manera que si antes no se había logrado establecer, formalmente, los objetos prioritarios de estudio de la ciencia política en el país, ahora se encuentran múltiples objetos de estudio muchas veces desvinculados de un tronco analítico común.

Frente a las distintas preocupaciones y problemas sobre el fenómeno de la política en Honduras, no se identifican escuelas teóricas que construyan argumentos, diseñen y apliquen metodologías para hacer consistentes las proposiciones. Más bien tiende a prevalecer en los analistas una opinión, que si bien parece lúcida, resalta más la agudeza intuitiva que la verificación empírica. Por el momento no se avizoran mayores signos de cambio en esta limitación, por cuanto existe una débil institucionalidad para la investigación politológica, sin perjuicio de que, como se mencionó antes, existen apoyos directos de varias agencias de cooperación internacional, aunque obviamente más dentro de la racionalidad de un análisis micropolítico o sectorial, pero poco dirigidos hacia estudios que permitan la formulación de proposiciones teóricas sobre la realidad política nacional, con el adecuado soporte empírico. En principio, la investigación básica que debería provenir de las universidades es en este campo exigua, con escasos recursos disponibles y en general condiciones adversas para la labor científica ${ }^{8}$.

Este inocultable déficit en la capacidad de construcción teórica no puede menos que servir de acicate para conjuntar esfuerzos en el país para insertarse en las corrientes politológicas regionales y sub-regionales, mediante el ingreso a redes científico-académicas, aprovechando al máximo los espacios de formación, actualización, investigación y discusión científica, a manera de formar una masa crítica que haga posible en Honduras la creación de bases consistentes de una ciencia política que esté a la altura de los tiempos.

\section{REFERENCIAS}

Garretón, Manuel Antonio. 1991. "Política, cultura y sociedad en la transición democrática". Revista Nueva Sociedad 114 (julio-agosto): 43-49.

Gomáriz, Enrique. 1996. La crisis de las ciencias sociales. Un Estudio Comparado. 1996. San José: FLACSO-Costa Rica. Lechner, Norbert. 1994. "Los nuevos perfiles de la política. Un Bosquejo". Revista Nueva Sociedad 130 (marzo-abril): 32-43. Orellana, Armando. 2005. Entrevista personal realizada por los autores. Tegucigalpa D.C., Honduras.

Paz, Ernesto. 2005. Entrevista personal realizada por los autores. Tegucigalpa D.C., Honduras.

Paz, Ernesto. 2004. EI Desarrollo de las Ciencias Políticas en Honduras. Ponencia presentada en el VI Congreso Centroamericano de Historia realizado en Tegucigalpa Honduras. Julio de 2004. Documento mímeo.

8 Como resultado de esta situación, durante los años 80 y 90, no pocos académicos salieron de las universidades para insertarse en las ONG y las agencias de cooperación internacional, donde se contaba con mayores fondos para la investigación; aunque claro está, la investigación privilegiada por estas entidades responde a inquietudes por lo general de corto y medio plazo, y en menor medida a la necesidad de generar investigación aplicada para producir un cuerpo organizado de conocimiento científico en el campo de la política. En síntesis, fuera de las universidades se contaba con mayor apoyo para la investigación empírica, pero debido a las prioridades de estos organismos, el abordaje empírico no ha ido acompañado de teorización. 
Álvaro Cálix Rodríguez es licenciado en Ciencias Jurídicas y Sociales y Master en Administración de Empresas por la Universidad Nacional Autónoma de Honduras (UNAH). Actualmente es doctorante en Ciencias Sociales con orientación a la Gestión del Desarrollo, en el Programa Latinoamericano de Trabajo Social de la UNAH. Coordinó el Informe Nacional sobre Desarrollo Humano del año 2003, bajo el auspicio del PNUD. Ha escrito varios ensayos sobre el sistema electoral en Honduras, el funcionamiento del sistema de justicia, la emergencia de la sociedad civil en Honduras, la seguridad ciudadana y la legitimidad democrática, entre otros. Por estos días se desempeña como investigador social, y es consultor asociado a la Fundación Friedrich Ebert en Honduras.

(E-mail: alvarocalix2002@yahoo.com)

Rolando Sierra Fonseca es licenciado en Historia por la Universidad Nacional Autónoma de Honduras; Magíster en Sociología por la Universidad Academia de Humanismo Cristiano de Chile y Magíster en Ciencias Sociales y del Desarrollo por el ILADES/Universidad Gregoriana de Roma. Ha sido Coordinador de los Informes Nacionales sobre Desarrollo Humano en Honduras en el año 2000 y en el 2002, bajo el auspicio del PNUD. Ha sido profesor universitario y es autor de doce libros. También ha publicado varios artículos en el campo de la historia de Honduras, el desarrollo humano, procesos de modernización y desarrollo de las ciencias sociales. Actualmente se desempeña como consultor en desarrollo y es consultor asociado al Centro de Investigación y Promoción de los Derechos Humanos en Honduras (CIPRODEH).

(E-mail: rsierrafonseca@unah.edu.hn ) 